\title{
Pieri's formula for generalized Schur polynomials
}

\author{
Yasuhide Numata
}

Received: 16 June 2006 / Accepted: 16 November 2006 /

Published online: 10 January 2007

(C) Springer Science + Business Media, LLC 2007

\begin{abstract}
Young's lattice, the lattice of all Young diagrams, has the RobinsonSchensted-Knuth correspondence, the correspondence between certain matrices and pairs of semi-standard Young tableaux with the same shape. Fomin introduced generalized Schur operators to generalize the Robinson-Schensted-Knuth correspondence. In this sense, generalized Schur operators are generalizations of semi-standard Young tableaux. We define a generalization of Schur polynomials as expansion coefficients of generalized Schur operators. We show that the commutation relation of generalized Schur operators implies Pieri's formula for generalized Schur polynomials.
\end{abstract}

Keywords Pieri formula $\cdot$ Generarized Schur operators $\cdot$ Schur polynomials $\cdot$ Young diagrams $\cdot$ Planar binary trees $\cdot$ Differential posets $\cdot$ Dual graphs $\cdot$ Symmetric functions · Quasi-symmetric polynomials

\section{Introduction}

Young's lattice is a prototypical example of a differential poset which was first defined by Stanley $[9,10]$. The Robinson correspondence is a correspondence between permutations and pairs of standard tableaux whose shapes are the same Young diagram. This correspondence was generalized for differential posets or dual graphs (generalizations of differential posets [3]) by Fomin [2, 4]. (See also [8].)

Young's lattice also has The Robinson-Schensted-Knuth correspondence, the correspondence between certain matrices and pairs of semi-standard tableaux. Fomin [5] introduced operators called generalized Schur operators, and generalized the Robinson-Schensted-Knuth correspondence to generalized Schur operators. We define

Y. Numata $(\bowtie)$

Department of Mathematics, Hokkaido University, Kita 10, Nishi 8, Kita-Ku, Sapporo, Hokkaido, 060-0810, Japan

e-mail: nu@math.sci.hokudai.ac.jp 
a generalization of Schur polynomials as expansion coefficients of generalized Schur operators.

A complete symmetric polynomial is a Schur polynomial associated with a Young diagram consisting of only one row. Schur polynomials satisfy Pieri's formula, the formula describing the product of a complete symmetric polynomial and a Schur polynomial as a sum of Schur polynomials:

$$
h_{i}\left(t_{1}, \ldots, t_{n}\right) s_{\lambda}\left(t_{1}, \ldots, t_{n}\right)=\sum_{\mu} s_{\mu}\left(t_{1}, \ldots, t_{n}\right)
$$

where the sum is over all $\mu$ 's that are obtained from $\lambda$ by adding $i$ boxes, with no two in the same column, $h_{i}$ is the $i$-th complete symmetric polynomial, and $s_{\lambda}$ is the Schur polynomial associated with $\lambda$.

In this paper, we generalize Pieri's formula to generalized Schur polynomials.

Remark 1.1. Lam introduced a generalization of the Boson-Fermion correspondence [6]. In the paper, he also showed Pieri's and Cauchy's formulae for some families of symmetric functions in the context of Heisenberg algebras. Some important families of symmetric functions, e.g., Schur functions, Hall-Littlewood polynomials, Macdonald polynomials and so on, are examples of them. He proved Pieri's formula using essentially the same method as the one in this paper. Since the assumptions of generalized Schur operators are less than those of Heisenberg algebras, our polynomials are more general than his; e.g., some of our polynomials are not symmetric. An example of generalized Schur operators which provides non-symmetric polynomials is in Section 4.3. See also Remark 2.8 for the relation between [6] and this paper.

This paper is organized as follows: In Section 2.1, we recall generalized Schur operators, and define generalized Schur polynomials. We also define a generalization of complete symmetric polynomials, called weighted complete symmetric polynomials, in Section 2.2. In Section 3, we show Pieri's formula for these polynomials (Theorem 3.2). We also see that Theorem 3.2 becomes simple for special parameters, and that weighted complete symmetric polynomials are written as linear combinations of generalized Schur polynomials in a special case. Other examples are shown in Section 4.

\section{Definition}

We introduce two types of polynomials in this section. One is a generalization of Schur polynomials. The other is a generalization of complete symmetric polynomials.

\subsection{Generalized schur polynomials}

First we recall the generalized Schur operators defined by Fomin [5]. We define a generalization of Schur polynomials as expansion coefficients of generalized Schur operators.

Let $K$ be a field of characteristic zero that contains all formal power series in variables $t, t^{\prime}, t_{1}, t_{2}, \ldots$ Let $V_{i}$ be finite-dimensional $K$-vector spaces for all $i \in \mathbb{Z}$. Fix 
a basis $Y_{i}$ of each $V_{i}$ so that $V_{i}=K Y_{i}$. Let $Y=\bigcup_{i} Y_{i}, V=\bigoplus_{i} V_{i}$ and $\widehat{V}=\prod_{i} V_{i}$, i.e., $V$ is the vector space consisting of all finite linear combinations of elements of $Y$ and $\widehat{V}$ is the vector space consisting of all linear combinations of elements of $Y$. The rankfunction on $V$ mapping $v \in V_{i}$ to $i$ is denoted by $\rho$. We say that $Y$ has a minimum $\varnothing$ if $Y_{i}=\varnothing$ for $i<0$ and $Y_{0}=\{\varnothing\}$.

For a sequence $\left\{A_{i}\right\}$ and a formal variable $x$, we write $A(x)$ for the generating function $\sum_{i \geq 0} A_{i} x^{i}$.

Definition 2.1. Let $D_{i}$ and $U_{i}$ be linear maps on $V$ for nonnegative integers $i \in \mathbb{N}$. We call $D\left(t_{1}\right) \cdots D\left(t_{n}\right)$ and $U\left(t_{n}\right) \cdots U\left(t_{1}\right)$ generalized Schur operators with $\left\{a_{m}\right\}$ if the following conditions are satisfied:

- $\left\{a_{m}\right\}$ is a sequence of $K$.

- $U_{i}$ satisfies $U_{i}\left(V_{j}\right) \subset V_{j+i}$ for all $j$.

- $D_{i}$ satisfies $D_{i}\left(V_{j}\right) \subset V_{j-i}$ for all $j$.

- The equation $D\left(t^{\prime}\right) U(t)=a\left(t t^{\prime}\right) U(t) D\left(t^{\prime}\right)$ holds.

In general, $D\left(t_{1}\right) \cdots D\left(t_{n}\right)$ and $U\left(t_{n}\right) \cdots U\left(t_{1}\right)$ are not linear operators on $V$ but linear operators from $V$ to $\widehat{V}$.

Let $\langle$,$\rangle be the natural pairing, i.e., the bilinear form on \widehat{V} \times V$ such that $\left\langle\sum_{\lambda \in Y} a_{\lambda} \lambda, \sum_{\mu \in Y} b_{\mu} \mu\right\rangle=\sum_{\lambda \in Y} a_{\lambda} b_{\lambda}$. For generalized Schur operators $D\left(t_{1}\right) \cdots$ $D\left(t_{n}\right)$ and $U\left(t_{n}\right) \cdots U\left(t_{1}\right), U_{i}^{*}$ and $D_{i}^{*}$ denote the maps obtained from the adjoints of $U_{i}$ and $D_{i}$ with respect to $\langle$,$\rangle by restricting to V$, respectively. For all $i, U_{i}^{*}$ and $D_{i}^{*}$ are linear maps on $V$ satisfying $U_{i}^{*}\left(V_{j}\right) \subset V_{j-i}$ and $D_{i}^{*}\left(V_{j}\right) \subset V_{j+i}$. It follows by definition that

$$
\left\langle v, U_{i} w\right\rangle=\left\langle w, U_{i}^{*} v\right\rangle, \quad\left\langle v, D_{i} w\right\rangle=\left\langle w, D_{i}^{*} v\right\rangle
$$

for $v, w \in V$. We write $U^{*}(t)$ and $D^{*}(t)$ for $\sum U_{i}^{*} t^{i}$ and $\sum D_{i}^{*} t^{i}$. It follows by definition that

$$
\langle U(t) \mu, \lambda\rangle=\left\langle U^{*}(t) \lambda, \mu\right\rangle, \quad\langle D(t) \mu, \lambda\rangle=\left\langle D^{*}(t) \lambda, \mu\right\rangle
$$

for $\lambda, \mu \in Y$. The equation $D\left(t^{\prime}\right) U(t)=a\left(t t^{\prime}\right) U(t) D\left(t^{\prime}\right)$ implies the equation $U^{*}\left(t^{\prime}\right) D^{*}(t)=a\left(t t^{\prime}\right) D^{*}(t) U^{*}\left(t^{\prime}\right)$. Hence $U^{*}\left(t_{1}\right) \cdots U^{*}\left(t_{n}\right)$ and $D^{*}\left(t_{n}\right) \cdots D^{*}\left(t_{1}\right)$ are generalized Schur operators with $\left\{a_{m}\right\}$ when $D\left(t_{1}\right) \cdots D\left(t_{n}\right)$ and $U\left(t_{n}\right) \cdots U\left(t_{1}\right)$ are.

Definition 2.2. Let $D\left(t_{1}\right) \cdots D\left(t_{n}\right)$ and $U\left(t_{n}\right) \cdots U\left(t_{1}\right)$ be generalized Schur operators with $\left\{a_{m}\right\}$. For $v \in V$ and $\mu \in Y, s_{v, \mu}^{D}\left(t_{1}, \ldots, t_{n}\right)$ and $s_{U}^{\mu, v}\left(t_{1}, \ldots, t_{n}\right)$ are respectively defined by

$$
\begin{aligned}
& s_{v, \mu}^{D}\left(t_{1}, \ldots, t_{n}\right)=\left\langle D\left(t_{1}\right) \cdots D\left(t_{n}\right) v, \mu\right\rangle, \\
& s_{U}^{\mu, v}\left(t_{1}, \ldots, t_{n}\right)=\left\langle U\left(t_{n}\right) \cdots U\left(t_{1}\right) v, \mu\right\rangle .
\end{aligned}
$$


We call these polynomials $s_{v, \mu}^{D}\left(t_{1}, \ldots, t_{n}\right)$ and $s_{U}^{\mu, v}\left(t_{1}, \ldots, t_{n}\right)$ generalized Schur polynomials.

Remark 2.3. Generalized Schur polynomials $s_{v, \mu}^{D}\left(t_{1}, \ldots, t_{n}\right)$ are symmetric in the case when $D(t) D\left(t^{\prime}\right)=D\left(t^{\prime}\right) D(t)$, but not symmetric in general. Similarly, generalized Schur polynomials $s_{U}^{\mu, v}\left(t_{1}, \ldots, t_{n}\right)$ are symmetric if $U(t) U\left(t^{\prime}\right)=U\left(t^{\prime}\right) U(t)$.

If $U_{0}$ (resp. $D_{0}$ ) is the identity map on $V$, generalized Schur polynomials $s_{v, \mu}^{D}\left(t_{1}, \ldots, t_{n}\right)$ (resp. $s_{U}^{\mu, v}\left(t_{1}, \ldots, t_{n}\right)$ ) are quasi-symmetric. In [1], Bergeron, Mykytiuk, Sottile and van Willigenburg considered graded representations of the algebra of noncommutative symmetric functions on the $\mathbb{Z}$-free module whose basis is a graded poset, and gave a Hopf-morphism from a Hopf algebra generated by intervals of the poset to the Hopf algebra of quasi-symmetric functions.

Example 2.4. Our prototypical example is Young's lattice $\mathbb{Y}$ that consists of all Young diagrams. Let $Y$ be Young's lattice $\mathbb{Y}, V$ the $K$-vector space $K \mathbb{Y}$ whose basis is $\mathbb{Y}$, and $\rho$ the ordinary rank function mapping a Young diagram $\lambda$ to the number of boxes in $\lambda$. Young's lattice $\mathbb{Y}$ has a minimum $\varnothing$, the Young diagram with no boxes. We call a skew Young diagram $\mu / \lambda$ a horizontal strip if $\mu / \lambda$ has no two boxes in the same column. Define $U_{i}$ by $U_{i}(\mu)=\sum_{\lambda} \lambda$, where the sum is over all $\lambda$ 's that are obtained from $\mu$ by adding a horizontal strip consisting of $i$ boxes; and define $D_{i}$ by $D_{i}(\lambda)=\sum_{\mu} \mu$, where the sum is over all $\mu$ 's that are obtained from $\lambda$ by removing a horizontal strip consisting of $i$ boxes. For example,

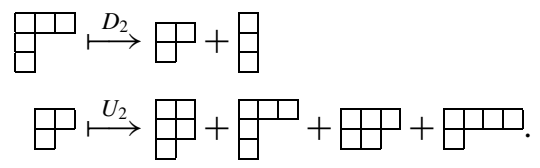

(See also Fig. 1, the graph of $D_{1}\left(U_{1}\right)$ and $D_{2}\left(U_{2}\right)$.)

In this case, $D\left(t_{1}\right) \cdots D\left(t_{n}\right)$ and $U\left(t_{n}\right) \cdots U\left(t_{1}\right)$ are generalized Schur operators with $\left\{a_{m}=1\right\}$. Both $s_{\lambda, \mu}^{D}\left(t_{1}, \ldots, t_{n}\right)$ and $s_{U}^{\lambda, \mu}\left(t_{1}, \ldots, t_{n}\right)$ are equal to the skew Schur

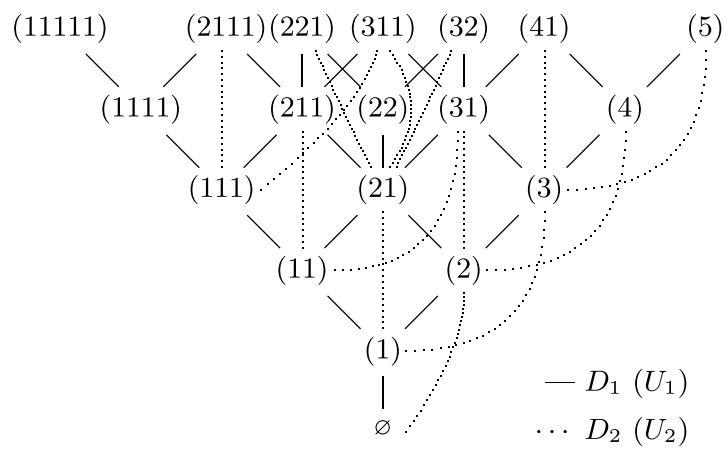

Fig. 1 Young's lattice

$\underline{\text { Springer }}$ 
polynomial $s_{\lambda / \mu}\left(t_{1}, \ldots, t_{n}\right)$ for $\lambda, \mu \in \mathbb{Y}$. For example, since

$$
\begin{aligned}
& D\left(t_{2}\right) \square=\square+t_{2} \square+t_{2} \square+t_{2}^{2} \square \\
& D\left(t_{1}\right) D\left(t_{2}\right) \square=\square+t_{1} \boxminus+t_{1} \square+t_{1}^{2} \square+t_{2}\left(\boxminus+t_{1} \square\right)+t_{2}\left(\square+t_{1} \square+t_{1}^{2} \varnothing\right) \\
& +t_{2}^{2}\left(\square+t_{1} \varnothing\right) \\
& s_{(2,1), \varnothing}^{D}\left(t_{1}, t_{2}\right)=s_{(2,1)}\left(t_{1}, t_{2}\right)=t_{1}^{2} t_{2}+t_{1} t_{2}^{2} .
\end{aligned}
$$

Example 2.5. Our second example is the polynomial ring $K[x]$ with a variable $x$. Let $V$ be $K[x]$ and $\rho$ the ordinary rank function mapping a monomial $a x^{n}$ to its degree $n$. In this case, $\operatorname{dim} V_{i}=1$ for all $i \geq 0$ and $\operatorname{dim} V_{i}=0$ for $i<0$. Hence its basis $Y$ is identified with $\mathbb{N}$ and has a minimum $c_{0}$, a nonzero constant. Define $D_{i}$ and $U_{i}$ by $\frac{\partial^{i}}{i !}$ and $\frac{x^{i}}{i !}$, where $\partial$ is the partial differential operator in $x$. Then $D(t)$ and $U(t)$ are $\exp (t \partial)$ and $\exp (t x)$. Since $D(t)$ and $U(t)$ satisfy $D(t) U\left(t^{\prime}\right)=\exp \left(t t^{\prime}\right) U\left(t^{\prime}\right) D(t)$, $D\left(t_{1}\right) \cdots D\left(t_{n}\right)$ and $U\left(t_{n}\right) \cdots U\left(t_{1}\right)$ are generalized Schur operators with $\left\{a_{m}=\frac{1}{m !}\right\}$. In general, for differential posets, we can construct generalized Schur operators in a similar manner.

Since $\partial$ and $x$ commute with $t$, the following equations hold:

$$
\begin{aligned}
D\left(t_{1}\right) \cdots D\left(t_{n}\right) & =\exp \left(\partial t_{1}\right) \cdots \exp \left(\partial t_{n}\right)=\exp \left(\partial\left(t_{1}+\cdots+t_{n}\right)\right) \\
U\left(t_{n}\right) \cdots U\left(t_{1}\right) & =\exp \left(x t_{n}\right) \cdots \exp \left(x t_{1}\right)=\exp \left(x\left(t_{1}+\cdots+t_{n}\right)\right) .
\end{aligned}
$$

It follows from direct calculations that

$$
\begin{aligned}
\exp \left(\partial\left(t_{1}+\cdots+t_{n}\right)\right) c_{i} x^{i} & =\sum_{j=0}^{i} \frac{\left(t_{1}+\cdots+t_{n}\right)^{j}}{j !} \frac{i !}{(i-j) !} c_{i} x^{i-j} \\
& =\sum_{j=0}^{i} \frac{i !\left(t_{1}+\cdots+t_{n}\right)^{j} c_{i}}{(i-j) ! j ! c_{i-j}} c_{i-j} x^{i-j}, \\
\exp \left(x\left(t_{1}+\cdots+t_{n}\right)\right) c_{i} x^{i} & =\sum_{j} \frac{\left(t_{1}+\cdots+t_{n}\right)^{j} x^{j}}{j !} c_{i} x^{i} \\
& =\sum_{j} \frac{\left(t_{1}+\cdots+t_{n}\right)^{j} c_{i}}{j ! c_{i+j}} c_{i+j} x^{i+j} .
\end{aligned}
$$

Hence it follows that

$$
\begin{aligned}
& s_{c_{i+j} x^{i+j}, c_{i} x^{i}}^{D}\left(t_{1}, \ldots, t_{n}\right)=\frac{(i+j) !}{i ! j !} \frac{c_{i+j}}{c_{i}}\left(t_{1}+\cdots+t_{n}\right)^{j} \\
& s_{U}^{c_{i+j} x^{i+j}, c_{i} x^{i}}\left(t_{1}, \ldots, t_{n}\right)=\frac{1}{j !} \frac{c_{i}}{c_{i+j}}\left(t_{1}+\cdots+t_{n}\right)^{j},
\end{aligned}
$$

if we take $\left\{c_{i} x^{i}\right\}$ as the basis $Y$. 
If $c_{i}=1$ for all $i$, then $s_{x^{i+j}, x^{i}}^{D}\left(t_{1}, \ldots, t_{n}\right)=\frac{(i+j) !}{i ! j !}\left(t_{1}+\cdots+t_{n}\right)^{j}$, and $s_{U}^{x^{i+j}, x^{i}}\left(t_{1}\right.$, $\left.\ldots, t_{n}\right)=\frac{1}{j !}\left(t_{1}+\cdots+t_{n}\right)^{j}$.

Lemma 2.6. Generalized Schur polynomials satisfy the following equations:

$$
\begin{aligned}
& s_{\lambda, \mu}^{D}\left(t_{1}, \ldots, t_{n}\right)=s_{D^{*}}^{\lambda, \mu}\left(t_{1}, \ldots, t_{n}\right), \\
& s_{U}^{\lambda, \mu}\left(t_{1}, \ldots, t_{n}\right)=s_{\lambda, \mu}^{U^{*}}\left(t_{1}, \ldots, t_{n}\right)
\end{aligned}
$$

for $\lambda, \mu \in Y$. Generalized Schur polynomials also satisfy the following equations:

$$
\begin{aligned}
s_{v, \mu}^{D}\left(t_{1}, \ldots, t_{n}\right) & =\sum_{v \in Y}\langle v, v\rangle s_{D^{*}}^{v, \mu}\left(t_{1}, \ldots, t_{n}\right), \\
s_{D^{*}}^{\mu, v}\left(t_{1}, \ldots, t_{n}\right) & =\sum_{v \in Y}\langle v, v\rangle s_{\mu, v}^{D}\left(t_{1}, \ldots, t_{n}\right), \\
s_{v, \mu}^{U^{*}}\left(t_{1}, \ldots, t_{n}\right) & =\sum_{v \in Y}\langle v, v\rangle s_{U}^{v, \mu}\left(t_{1}, \ldots, t_{n}\right), \\
s_{U}^{\mu, v}\left(t_{1}, \ldots, t_{n}\right) & =\sum_{v \in Y}\langle v, v\rangle s_{\mu, v}^{U^{*}}\left(t_{1}, \ldots, t_{n}\right)
\end{aligned}
$$

for $\mu \in Y, v \in V$.

Proof: It follows by definition that

$$
\begin{aligned}
s_{\lambda, \mu}^{D}\left(t_{1}, \ldots, t_{n}\right) & =\left\langle D\left(t_{1}\right) \cdots D\left(t_{n}\right) \lambda, \mu\right\rangle \\
& =\left\langle D^{*}\left(t_{n}\right) \cdots D^{*}\left(t_{1}\right) \mu, \lambda\right\rangle=s_{D^{*}}^{\lambda, \mu}\left(t_{1}, \ldots, t_{n}\right) .
\end{aligned}
$$

Similarly, we have $s_{U}^{\lambda, \mu}\left(t_{1}, \ldots, t_{n}\right)=s_{\lambda, \mu}^{U^{*}}\left(t_{1}, \ldots, t_{n}\right)$. The other formulae follow from $v=\sum_{v \in Y}\langle v, v\rangle v$ for $v \in V$.

Remark 2.7. Rewriting the generalized Cauchy identity [5, 1.4. Corollary] with our notation, we obtain a Cauchy identity for generalized Schur polynomials:

$$
\sum_{\nu \in Y} s_{v, \mu}^{D}\left(t_{1}, \ldots, t_{n}\right) s_{U}^{v, v}\left(t_{1}^{\prime}, \ldots, t_{n}^{\prime}\right)=\prod_{i, j} a\left(t_{i} t_{j}^{\prime}\right) \sum_{\kappa \in Y} s_{U}^{\mu, \kappa}\left(t_{1}^{\prime}, \ldots, t_{n}^{\prime}\right) s_{v, \kappa}^{D}\left(t_{1}, \ldots, t_{n}\right)
$$

for $v \in V, \mu \in Y$.

Remark 2.8. In this remark, we construct operators $B_{l}$ from generalized Schur operators $D\left(t_{1}\right) \cdots D\left(t_{n}\right)$ and $U\left(t_{n}\right) \cdots U\left(t_{1}\right)$. These operators $B_{l}$ are closely related to the results of Lam [6]. Furthermore we can construct other generalized Schur operators $D\left(t_{1}\right) \cdots D\left(t_{n}\right)$ and $U^{\prime}\left(t_{n}\right) \cdots U^{\prime}\left(t_{1}\right)$ from $B_{l}$.

Let $D\left(t_{1}\right) \cdots D\left(t_{n}\right)$ and $U\left(t_{n}\right) \cdots U\left(t_{1}\right)$ be generalized Schur operators with $\left\{a_{m}\right\}$. For a partition $\lambda \vdash l$, we define $z_{\lambda}$ by $z_{\lambda}=1^{m_{1}(\lambda)} m_{1}(\lambda) ! \cdot 2^{m_{2}(\lambda)} m_{2}(\lambda) ! \cdots$, where $\underline{\text { Springer }}$ 
$m_{i}(\lambda)=\left|\left\{j \mid \lambda_{j}=i\right\}\right|$. Let $U_{0}=D_{0}=I$, where $I$ is the identity map. For positive integers $l$, we inductively define $b_{l}, B_{l}$ and $B_{-l}$ by

$$
\begin{gathered}
b_{l}=a_{l}-\sum_{\lambda} \frac{b_{\lambda}}{z_{\lambda}}, \\
B_{l}=D_{l}-\sum_{\lambda} \frac{B_{\lambda}}{z_{\lambda}}, \\
B_{-l}=U_{l}-\sum_{\lambda} \frac{B_{-\lambda}}{z_{\lambda}},
\end{gathered}
$$

where $b_{\lambda}=b_{\lambda_{1}} \cdot b_{\lambda_{2}} \cdots, B_{\lambda}=B_{\lambda_{1}} \cdot B_{\lambda_{2}} \cdots, B_{-\lambda}=B_{-\lambda_{1}} \cdot B_{-\lambda_{2}} \cdots$ and the sums are over all partitions $\lambda$ of $l$ such that $\lambda_{1}<l$. Let $b_{l} \neq 0$ for any $l$. It follows from direct calculations that

$$
\begin{aligned}
& {\left[B_{l}, B_{-l}\right]=l \cdot b_{l} \cdot I,} \\
& {\left[B_{l}, B_{-k}\right]=0}
\end{aligned}
$$

for positive integers $l \neq k$. If $U_{i}$ and $D_{i}$ respectively commute with $U_{j}$ and $D_{j}$ for all $i, j$, then $\left\{B_{l}, B_{-l} \mid l \in \mathbb{Z}_{>0}\right\}$ generates the Heisenberg algebra. In this case, we can apply the results of Lam [6]. See also Remark 2.13 for the relation between his complete symmetric polynomials $h_{i}\left[b_{m}\right]\left(t_{1}, \ldots, t_{n}\right)$ and our weighted complete symmetric polynomials $h_{i}^{\left\{a_{m}\right\}}\left(t_{1}, \ldots, t_{n}\right)$.

For a partition $\lambda \vdash l$, let $\operatorname{sgn}(\lambda)$ denote $(-1)^{\sum_{i}\left(\lambda_{i}-1\right)}$, where the sum is over all $i$ 's such that $\lambda_{i}>0$. Although $U_{i}$ and $D_{i}$ do not commute with $U_{j}$ and $D_{j}$, we can define dual generalized Schur operators $D\left(t_{1}\right) \cdots D\left(t_{n}\right)$ and $U^{\prime}\left(t_{n}\right) \cdots U^{\prime}\left(t_{1}\right)$ with $\left\{a_{m}^{\prime}\right\}$ by

$$
\begin{aligned}
a_{l}^{\prime} & =\sum_{\lambda} \frac{\operatorname{sgn}(\lambda) b_{\lambda}}{z_{\lambda}}, \\
U_{-l}^{\prime} & =\sum_{\lambda} \frac{\operatorname{sgn}(\lambda) B_{-\lambda}}{z_{\lambda}},
\end{aligned}
$$

where the sums are over all partitions $\lambda$ of $l$. In this case, it follows from direct calculations that $a(t) \cdot a^{\prime}(-t)=1$.

\subsection{Weighted complete symmetric polynomials}

Next we introduce a generalization of complete symmetric polynomials. We define weighted symmetric polynomials inductively.

Definition 2.9. Let $\left\{a_{m}\right\}$ be a sequence of elements of $K$. We define the $i$-th weighted complete symmetric polynomial $h_{i}^{\left\{a_{m}\right\}}\left(t_{1}, \ldots, t_{n}\right)$ to be the coefficient of $t^{i}$ in $a\left(t_{1} t\right) \cdots a\left(t_{n} t\right)$. 
By definition, for each $i$, the $i$-th weighted complete symmetric polynomial $h_{i}^{\left\{a_{m}\right\}}\left(t_{1}, \ldots, t_{n}\right)$ is a homogeneous symmetric polynomial of degree $i$.

Remark 2.10. For a sequence $\left\{a_{m}\right\}$ of elements of $K$, the $i$-th weighted complete symmetric polynomial $h_{i}^{\left\{a_{m}\right\}}\left(t_{1}, \ldots, t_{n}\right)$ coincides with the polynomial defined by

$$
h_{i}^{\left\{a_{m}\right\}}\left(t_{1}, \ldots, t_{n}\right)= \begin{cases}a_{i} t_{1}^{i} & (\text { for } n=1), \\ \sum_{j=0}^{i} h_{j}^{\left\{a_{m}\right\}}\left(t_{1}, \ldots, t_{n-1}\right) h_{i-j}^{\left\{a_{m}\right\}}\left(t_{n}\right) & (\text { for } n>1) .\end{cases}
$$

Example 2.11. When $a_{m}$ equals 1 for each $m, a(t)=\sum_{i} t^{i}=\frac{1}{1-t}$. In this case, $h_{j}^{\{1,1, \ldots\}}\left(t_{1}, \ldots, t_{n}\right)$ equals the complete symmetric polynomial $h_{j}\left(t_{1}, \ldots, t_{n}\right)$.

Example 2.12. When $a_{m}$ equals $\frac{1}{m !}$ for each $m, \sum_{j} h_{j}^{\left\{\frac{1}{m !}\right\}}(t)=\exp (t)=a(t)$ and $h_{j}^{\left\{\frac{1}{m !}\right\}}\left(t_{1}, \ldots, t_{n}\right)=\frac{1}{j !}\left(t_{1}+\cdots+t_{n}\right)^{j}$.

Remark 2.13. In this remark, we compare the complete symmetric polynomials $h_{i}\left[b_{m}\right]\left(t_{1}, \ldots, t_{n}\right)$ of Lam [6] and our weighted complete symmetric polynomials $h_{i}^{\left\{a_{m}\right\}}\left(t_{1}, \ldots, t_{n}\right)$. Let $\left\{b_{m}\right\}$ be a sequence of elements of $K$. The polynomials $h_{i}\left[b_{m}\right]\left(t_{1}, \ldots, t_{n}\right)$ of Lam are defined by

$$
h_{i}\left[b_{m}\right]\left(t_{1}, \ldots, t_{n}\right)=\sum_{\lambda \vdash i} \frac{b_{\lambda} p_{\lambda}\left(t_{1}, \ldots, t_{n}\right)}{z_{\lambda}},
$$

where $b_{\lambda}=b_{\lambda_{1}} \cdot b_{\lambda_{2}} \cdots, \quad p_{\lambda}\left(t_{1}, \ldots, t_{n}\right)=p_{\lambda_{1}}\left(t_{1}, \ldots, t_{n}\right) \cdot p_{\lambda_{2}}\left(t_{1}, \ldots, t_{n}\right) \cdots \quad$ and $p_{i}\left(t_{1}, \ldots, t_{n}\right)=t_{1}^{i}+\cdots+t_{n}^{i}$. These polynomials satisfy the equation

$$
h_{i}\left[b_{m}\right]\left(t_{1}, \ldots, t_{n}\right)=\sum_{j=0}^{i} h_{j}\left[b_{m}\right]\left(t_{1}, \ldots, t_{n-1}\right) h_{i-j}\left[b_{m}\right]\left(t_{n}\right) .
$$

Let $a_{i}=\sum_{\lambda \vdash i} \frac{b_{\lambda}}{z_{\lambda}}$. Then it follows $h_{i}\left[b_{m}\right]\left(t_{1}\right)=a_{i} t^{i}$. Hence

$$
h_{i}\left[b_{m}\right]\left(t_{1}, \ldots, t_{n}\right)=h_{i}^{\left\{a_{m}\right\}}\left(t_{1}, \ldots, t_{n}\right)
$$

\section{Main results}

In this section, we show some properties of generalized Schur polynomials and weighted complete symmetric polynomials.

Throughout this section, let $D\left(t_{1}\right) \cdots D\left(t_{n}\right)$ and $U\left(t_{n}\right) \cdots U\left(t_{1}\right)$ be generalized Schur operators with $\left\{a_{m}\right\}$. 


\subsection{Main theorem}

In Proposition 3.1, we describe the commuting relation of $U_{i}$ and $D\left(t_{1}\right) \cdots D\left(t_{n}\right)$, proved in Section 3.3. This relation implies Pieri's formula for our polynomials (Theorem 3.2), the main result in this paper. It also follows from this relation that the weighted complete symmetric polynomials are written as linear combinations of generalized Schur polynomials when $Y$ has a minimum (Proposition 3.5).

First we describe the commuting relation of $U_{i}$ and $D\left(t_{1}\right) \cdots D\left(t_{n}\right)$. We prove it in Section 3.3.

Proposition 3.1. The equations

$$
\begin{aligned}
D\left(t_{1}\right) \cdots D\left(t_{n}\right) U_{i} & =\sum_{j=0}^{i} h_{i-j}^{\left\{a_{m}\right\}}\left(t_{1}, \ldots, t_{n}\right) U_{j} D\left(t_{1}\right) \cdots D\left(t_{n}\right), \\
D_{i} U\left(t_{n}\right) \cdots U\left(t_{1}\right) & =\sum_{j=0}^{i} h_{i-j}^{\left\{a_{m}\right\}}\left(t_{1}, \ldots, t_{n}\right) U\left(t_{n}\right) \cdots U\left(t_{1}\right) D_{j}, \\
U_{i}^{*} D^{*}\left(t_{n}\right) \cdots D^{*}\left(t_{1}\right) & =\sum_{j=0}^{i} h_{i-j}^{\left\{a_{m}\right\}}\left(t_{1}, \ldots, t_{n}\right) D^{*}\left(t_{n}\right) \cdots D^{*}\left(t_{1}\right) U_{j}^{*}, \\
U^{*}\left(t_{1}\right) \cdots U^{*}\left(t_{n}\right) D_{i}^{*} & =\sum_{j=0}^{i} h_{i-j}^{\left\{a_{m}\right\}}\left(t_{1}, \ldots, t_{n}\right) D_{j}^{*} U^{*}\left(t_{1}\right) \cdots U^{*}\left(t_{n}\right) .
\end{aligned}
$$

hold for all $i$.

These equations imply the following main theorem.

Theorem 3.2 (Pieri's formula). For each $\mu \in Y_{k}$ and each $v \in V$, generalized Schur polynomials satisfy

$$
s_{U_{i} v, \mu}^{D}\left(t_{1}, \ldots, t_{n}\right)=\sum_{j=0}^{i} h_{i-j}^{\left\{a_{m}\right\}}\left(t_{1}, \ldots, t_{n}\right) \sum_{\nu \in Y_{k-j}}\left\langle U_{j} v, \mu\right\rangle s_{v, v}^{D}\left(t_{1}, \ldots, t_{n}\right) .
$$

Proof: It follows from Proposition 3.1 that

$$
\begin{aligned}
\left\langle D\left(t_{1}\right) \cdots D\left(t_{n}\right) U_{i} v, \mu\right\rangle & =\left\langle\sum_{j=0}^{i} h_{i-j}^{\left\{a_{m}\right\}}\left(t_{1}, \ldots, t_{n}\right) U_{j} D\left(t_{1}\right) \cdots D\left(t_{n}\right) v, \mu\right\rangle \\
& =\sum_{j=0}^{i} h_{i-j}^{\left\{a_{m}\right\}}\left(t_{1}, \ldots, t_{n}\right)\left\langle U_{j} D\left(t_{1}\right) \cdots D\left(t_{n}\right) v, \mu\right\rangle
\end{aligned}
$$


for $v \in V$ and $\mu \in Y$. This says

$$
s_{U_{i} v, \mu}^{D}\left(t_{1}, \ldots, t_{n}\right)=\sum_{j=0}^{i} h_{i-j}^{\left\{a_{m}\right\}}\left(t_{1}, \ldots, t_{n}\right) \sum_{\nu \in Y_{k-j}}\left\langle U_{j} v, \mu\right\rangle s_{v, v}^{D}\left(t_{1}, \ldots, t_{n}\right) .
$$

This formula becomes simple in the case when $v \in Y$.

Corollary 3.3. For each $\lambda, \mu \in Y$, generalized Schur polynomials satisfy

$$
s_{U_{i} \lambda, \mu}^{D}\left(t_{1}, \ldots, t_{n}\right)=\sum_{j=0}^{i} h_{i-j}^{\left\{a_{m}\right\}}\left(t_{1}, \ldots, t_{n}\right) \cdot s_{D^{*}}^{\lambda, U_{j}^{*} \mu}\left(t_{1}, \ldots, t_{n}\right) .
$$

Proof: It follows from Theorem 3.2 that

$$
s_{U_{i} \lambda, \mu}^{D}\left(t_{1}, \ldots, t_{n}\right)=\sum_{j=0}^{i} h_{i-j}^{\left\{a_{m}\right\}}\left(t_{1}, \ldots, t_{n}\right) \sum_{\nu \in Y}\left\langle U_{j} \nu, \mu\right\rangle s_{\lambda, \nu}^{D}\left(t_{1}, \ldots, t_{n}\right) .
$$

Lemma 2.6 implies

$$
\begin{aligned}
\sum_{\nu \in Y}\left\langle U_{j} v, \mu\right\rangle s_{\lambda, v}^{D}\left(t_{1}, \ldots, t_{n}\right) & =\sum_{v \in Y}\left\langle v, U_{j}^{*} \mu\right\rangle s_{\lambda, v}^{D}\left(t_{1}, \ldots, t_{n}\right) \\
& =s_{D^{*}}^{\lambda, U_{j}^{*} \mu}\left(t_{1}, \ldots, t_{n}\right) .
\end{aligned}
$$

Hence

$$
s_{U_{i} \lambda, \mu}^{D}\left(t_{1}, \ldots, t_{n}\right)=\sum_{j=0}^{i} h_{i-j}^{\left\{a_{m}\right\}}\left(t_{1}, \ldots, t_{n}\right) \cdot s_{D^{*}}^{\lambda, U_{j}^{*} \mu}\left(t_{1}, \ldots, t_{n}\right) .
$$

If $Y$ has a minimum $\varnothing$, Theorem 3.2 implies the following corollary.

Corollary 3.4. Let $Y$ have a minimum $\varnothing$. For all $v \in V$, the following equations hold:

$$
s_{U_{i} v, \varnothing}^{D}\left(t_{1}, \ldots, t_{n}\right)=u_{0} \cdot h_{i}^{\left\{a_{m}\right\}}\left(t_{1}, \ldots, t_{n}\right) \cdot s_{v, \varnothing}^{D}\left(t_{1}, \ldots, t_{n}\right),
$$

where $u_{0}$ is the element of $K$ that satisfies $U_{0} \varnothing=u_{0} \varnothing$.

In the case when $Y$ has a minimum $\varnothing$, weighted complete symmetric polynomials are written as linear combinations of generalized Schur polynomials.

Proposition 3.5. Let $Y$ have a minimum $\varnothing$. The following equations hold for all $i \geq 0$ :

$$
s_{U_{i} \varnothing, \varnothing}^{D}\left(t_{1}, \ldots, t_{n}\right)=d_{0}^{n} u_{0} \cdot h_{i}^{\left\{a_{m}\right\}}\left(t_{1}, \ldots, t_{n}\right),
$$


where $d_{0}, u_{0}$ are the elements of $K$ that satisfy $D_{0} \varnothing=d_{0} \varnothing$ and $U_{0} \varnothing=u_{0} \varnothing$.

Proof: By definition, $s_{\varnothing, \varnothing}^{D}\left(t_{1}, \ldots, t_{n}\right)$ is $d_{0}^{n}$. Hence it follows from Corollary 3.4 that

$$
s_{U_{i} \varnothing, \varnothing}^{D}\left(t_{1}, \ldots, t_{n}\right)=u_{0} h_{i}^{\left\{a_{m}\right\}}\left(t_{1}, \ldots, t_{n}\right) d_{0}^{n} .
$$

Example 3.6. In the prototypical example $\mathbb{Y}$ (Example 2.4), for $\lambda \in \mathbb{Y}, U_{i} \lambda$ is the sum of all Young diagrams obtained from $\lambda$ by adding a horizontal strip consisting of $i$ boxes. Hence $s_{U_{i} \lambda, \varnothing}^{D}\left(t_{1}, \ldots, t_{n}\right)$ equals $\sum_{v} s_{v}$, where the sum is over all $v$ 's that are obtained from $\lambda$ by adding a horizontal strip consisting of $i$ boxes. On the other hand, $u_{0}$ is 1 , and $h_{i}^{\{1,1,1, \ldots\}}\left(t_{1}, \ldots, t_{n}\right)$ is the $i$-th complete symmetric polynomial $h_{i}\left(t_{1}, \ldots, t_{n}\right)$ (Example 2.11). Thus Corollary 3.4 is nothing but the classical Pieri's formula. Theorem 3.2 is Pieri's formula for skew Schur polynomials; for a skew Young diagram $\lambda / \mu$ and $i \in \mathbb{N}$,

$$
\sum_{\kappa} s_{\kappa / \mu}\left(t_{1}, \ldots, t_{n}\right)=\sum_{j=0}^{i} \sum_{\nu} h_{i-j}\left(t_{1}, \ldots, t_{n}\right) s_{\lambda / v}\left(t_{1}, \ldots, t_{n}\right),
$$

where the first sum is over all $\kappa$ 's that are obtained from $\lambda$ by adding a horizontal strip consisting of $i$ boxes; the last sum is over all $\nu$ 's that are obtained from $\mu$ by removing a horizontal strip consisting of $j$ boxes.

In this example, Proposition 3.5 says that the Schur polynomial $s_{(i)}$ corresponding to Young diagram with only one row equals the complete symmetric polynomial $h_{i}$.

Example 3.7. In the second example $\mathbb{N}$ (Example 2.5), Proposition 3.5 says that the constant term of $\exp \left(\partial\left(t_{1}+\cdots+t_{n}\right)\right) \cdot \frac{x^{i}}{i !}$ equals $\frac{\left(t_{1}+\cdots+t_{n}\right)^{i}}{i !}$.

\subsection{Some variations of Pieri's formula}

In this section, we show some variations of Pieri's formula for generalized Schur polynomials, i.e., we show Pieri's formula not only for $s_{\lambda, \mu}^{D}\left(t_{1}, \ldots, t_{n}\right)$ but also for $s_{U}^{\lambda, \mu}\left(t_{1}, \ldots, t_{n}\right), s_{D^{*}}^{\lambda, \mu}\left(t_{1}, \ldots, t_{n}\right)$ and $s_{\lambda, \mu}^{U^{*}}\left(t_{1}, \ldots, t_{n}\right)$.

Theorem 3.8 (Pieri's formula). For each $\mu \in Y_{k}$ and each $v \in V$, generalized Schur polynomials satisfy the following equations:

$$
\begin{aligned}
\sum_{\kappa \in Y}\left\langle D_{i} \kappa, \mu\right\rangle s_{U}^{\kappa, v}\left(t_{1}, \ldots, t_{n}\right) & =\sum_{j=0}^{i} h_{i-j}^{\left\{a_{m}\right\}}\left(t_{1}, \ldots, t_{n}\right) s_{U}^{\mu, D_{j} v}\left(t_{1}, \ldots, t_{n}\right), \\
s_{D_{i}^{*} v, \mu}^{U^{*}}\left(t_{1}, \ldots, t_{n}\right) & =\sum_{j=0}^{i} h_{i-j}^{\left\{a_{m}\right\}}\left(t_{1}, \ldots, t_{n}\right) \sum_{v \in Y_{k-j}}\left\langle D_{j}^{*} v, \mu\right\rangle s_{v, v}^{U^{*}}\left(t_{1}, \ldots, t_{n}\right), \\
\sum_{\kappa \in Y}\left\langle U_{i}^{*} \kappa, \mu\right\rangle s_{D^{*}}^{\kappa, v}\left(t_{1}, \ldots, t_{n}\right) & =\sum_{j=0}^{i} h_{i-j}^{\left\{a_{m}\right\}}\left(t_{1}, \ldots, t_{n}\right) s_{D^{*}}^{\mu, U_{j}^{*} v}\left(t_{1}, \ldots, t_{n}\right) .
\end{aligned}
$$


Proof: Applying Theorem 3.2 to $U^{*}\left(t_{1}\right) \cdots U^{*}\left(t_{n}\right)$ and $D^{*}\left(t_{n}\right) \cdots D^{*}\left(t_{1}\right)$, we obtain

$$
s_{D_{i}^{*} v, \mu}^{U^{*}}\left(t_{1}, \ldots, t_{n}\right)=\sum_{j=0}^{i} h_{i-j}^{\left\{a_{m}\right\}}\left(t_{1}, \ldots, t_{n}\right) \sum_{\nu \in Y_{k-j}}\left\langle D_{j}^{*} v, \mu\right\rangle s_{v, \nu}^{U^{*}}\left(t_{1}, \ldots, t_{n}\right) .
$$

It follows from Proposition 3.1 that

$$
\left\langle D_{i} U\left(t_{n}\right) \cdots U\left(t_{1}\right) v, \mu\right\rangle=\left\langle\sum_{j=0}^{i} h_{i-j}^{\left\{a_{m}\right\}}\left(t_{1}, \ldots, t_{n}\right) U\left(t_{n}\right) \cdots U\left(t_{1}\right) D_{j} v, \mu\right\rangle
$$

for $v \in V$ and $\mu \in Y$. This equation says

$$
\sum_{\kappa \in Y}\left\langle D_{i} \kappa, \mu\right\rangle s_{U}^{\kappa, v}\left(t_{1}, \ldots, t_{n}\right)=\sum_{j=0}^{i} h_{i-j}^{\left\{a_{m}\right\}}\left(t_{1}, \ldots, t_{n}\right) s_{U}^{\mu, D_{j} v}\left(t_{1}, \ldots, t_{n}\right) .
$$

For generalized Schur operators $U^{*}\left(t_{1}\right) \cdots U^{*}\left(t_{n}\right)$ and $D^{*}\left(t_{n}\right) \cdots D^{*}\left(t_{1}\right)$, this equation says

$$
\sum_{\kappa \in Y}\left\langle U_{i}^{*} \kappa, \mu\right\rangle s_{D^{*}}^{\kappa, v}\left(t_{1}, \ldots, t_{n}\right)=\sum_{j=0}^{i} h_{i-j}^{\left\{a_{m}\right\}}\left(t_{1}, \ldots, t_{n}\right) s_{D^{*}}^{\mu, U_{j}^{*} v}\left(t_{1}, \ldots, t_{n}\right) .
$$

Corollary 3.9. For all $v \in V$, the following equations hold:

$$
s_{D_{i}^{*} v, \varnothing}^{U^{*}}\left(t_{1}, \ldots, t_{n}\right)=d_{0} \cdot h_{i}^{\left\{a_{m}\right\}}\left(t_{1}, \ldots, t_{n}\right) \cdot s_{v, \varnothing}^{U^{*}}\left(t_{1}, \ldots, t_{n}\right),
$$

where $d_{0}$ is the element of $K$ that satisfies $D_{0} \varnothing=d_{0} \varnothing$.

Proof: We obtain this proposition from Theorem 3.4 by applying to generalized Schur operators $U^{*}\left(t_{1}, \ldots, t_{n}\right)$ and $D^{*}\left(t_{1}, \ldots, t_{n}\right)$.

Proposition 3.10. Let $Y$ have a minimum $\varnothing$. Then

$$
s_{D_{i}^{*} \varnothing, \varnothing}^{U^{*}}\left(t_{1}, \ldots, t_{n}\right)=u_{0}^{n} d_{0} \cdot h_{i}^{\left\{a_{m}\right\}}\left(t_{1}, \ldots, t_{n}\right),
$$

where $u_{0}$ and $d_{0}$ are the elements of $K$ that satisfy $D_{0} \varnothing=d_{0} \varnothing$ and $U_{0} \varnothing=u_{0} \varnothing$.

Proof: We obtain this proposition by applying Theorem 3.5 to generalized Schur operators $U^{*}\left(t_{1}\right) \cdots U^{*}\left(t_{n}\right)$ and $D^{*}\left(t_{n}\right) \cdots D^{*}\left(t_{1}\right)$.

\subsection{Proof of Proposition 3.1}

In this section, we prove Proposition 3.1.

First, we prove the Eq. (2). The other equations follow from the Eq. (2). $\underline{\text { Springer }}$ 
Proof: Since $D\left(t_{1}\right) \cdots D\left(t_{n}\right)$ and $U\left(t_{n}\right) \cdots U\left(t_{1}\right)$ are generalized Schur operators with $\left\{a_{m}\right\}$, the equations $D(t) U_{i}=\sum_{j=0}^{i} a_{j} t^{j} U_{i-j} D(t)$ hold for all integers $i$. Hence $D\left(t_{1}\right) \cdots D\left(t_{n}\right) U_{i}$ is written as a $K$-linear combination of $U_{j} D\left(t_{1}\right) \cdots D\left(t_{n}\right)$. We write $H_{i, j}\left(t_{1}, \ldots, t_{n}\right)$ for the coefficient of $U_{j} D\left(t_{1}\right) \cdots D\left(t_{n}\right)$ in $D\left(t_{1}\right) \cdots D\left(t_{n}\right) U_{i}$.

It follows from the equation $D(t) U_{i}=\sum_{j=0}^{i} a_{j} t^{j} U_{i-j} D(t)$ that

$$
H_{i, i-j}\left(t_{1}\right)=a_{j} t_{1}^{j}
$$

for $0 \leq j \leq i$.

We apply the relation (6) to $D\left(t_{n}\right)$ and $U_{i}$ to have

$$
D\left(t_{1}\right) \cdots D\left(t_{n-1}\right) D\left(t_{n}\right) U_{i}=\sum_{j=0}^{i} a_{i-j} t_{n}^{i-j} D\left(t_{1}\right) \cdots D\left(t_{n-1}\right) U_{j} D\left(t_{n}\right) .
$$

Since $D\left(t_{1}\right) \cdots D\left(t_{n-1}\right) U_{i}=\sum_{j} H_{i, j}\left(t_{1}, \ldots, t_{n-1}\right) U_{j} D\left(t_{1}\right) \cdots D\left(t_{n-1}\right)$ by the definition of $H_{i, j}\left(t_{1}, \ldots, t_{n-1}\right)$, we have the equation

$$
D\left(t_{1}\right) \cdots D\left(t_{n-1}\right) D\left(t_{n}\right) U_{i}=\sum_{k=0}^{i} \sum_{j=k}^{i} a_{i-j} t_{n}^{i-j} H_{j, k}\left(t_{1}, \ldots, t_{n-1}\right) U_{k} D\left(t_{1}\right) \cdots D\left(t_{n}\right)
$$

Since $D\left(t_{1}\right) \cdots D\left(t_{n}\right) U_{i}$ equals $\sum_{k=0}^{i} H_{i, k}\left(t_{1}, \ldots, t_{n}\right) U_{k} D\left(t_{1}\right) \cdots D\left(t_{n}\right)$ by definition, the equation

$$
\begin{aligned}
& \sum_{k=0}^{i} \sum_{j=k}^{i} a_{i-j} t_{n}^{i-j} H_{j, k}\left(t_{1}, \ldots, t_{n-1}\right) U_{k} D\left(t_{1}\right) \cdots D\left(t_{n}\right) \\
& \quad=\sum_{k=0}^{i} H_{i, k}\left(t_{1}, \ldots, t_{n}\right) U_{k} D\left(t_{1}\right) \cdots D\left(t_{n}\right)
\end{aligned}
$$

holds. Hence the equation

$$
\sum_{j=k}^{i} a_{i-j} t_{n}^{i-j} H_{j, k}\left(t_{1}, \ldots, t_{n-1}\right)=H_{i, k}\left(t_{1}, \ldots, t_{n}\right)
$$

holds.

We claim that $H_{i+k, k}\left(t_{1}, \ldots, t_{n}\right)$ does not depend on $k$. It follows from this relation (7) that

$$
\begin{aligned}
H_{k+l, k}\left(t_{1}, \ldots, t_{n}\right) & =\sum_{j=k}^{k+l} a_{k+l-j} t_{n}^{k+l-j} H_{j, k}\left(t_{1}, \ldots, t_{n-1}\right) \\
& =\sum_{j^{\prime}=0}^{l} a_{k+l-\left(j^{\prime}+k\right)} t_{n}^{k+l-\left(j^{\prime}+k\right)} H_{j^{\prime}+k, k}\left(t_{1}, \ldots, t_{n-1}\right) \\
& =\sum_{j^{\prime}=0}^{l} a_{l-j^{\prime}} t_{n}^{l-j^{\prime}} H_{j^{\prime}+k, k}\left(t_{1}, \ldots, t_{n-1}\right) .
\end{aligned}
$$


Since the monomials $a_{l-j^{\prime}} t_{n}^{l-j^{\prime}}$ do not depend on $k$, the equations

$$
H_{(i-k)+k, k}\left(t_{1}, \ldots, t_{n}\right)=H_{(i-k)+k^{\prime}, k^{\prime}}\left(t_{1}, \ldots, t_{n}\right)
$$

hold if the equations $H_{k+j, k}\left(t_{1}, \ldots, t_{n-1}\right)=H_{k^{\prime}+j, k^{\prime}}\left(t_{1}, \ldots, t_{n-1}\right)$ hold for all $k, k^{\prime}$ and $j \leq i-k$. In fact, since $H_{i+k, k}\left(t_{1}\right)$ equals $a_{i} t_{1}^{i}, H_{i+k, k}\left(t_{1}\right)$ does not depend on $k$. Hence it follows inductively that $H_{i+k, k}\left(t_{1}, \ldots, t_{n}\right)$ does not depend on $k$, either. Hence we may write $\tilde{H}_{i-j}\left(t_{1}, \ldots, t_{n}\right)$ for $H_{i, j}\left(t_{1}, \ldots, t_{n}\right)$.

It follows from the Eqs. (6) and (7) that

$$
\begin{cases}\tilde{H}_{i}\left(t_{1}\right)=a_{i} t_{1}^{i} & (\text { for } n=1) \\ \tilde{H}_{i}\left(t_{1}, \ldots, t_{n}\right)=\sum_{k=0}^{i} \tilde{H}_{i-k}\left(t_{1}, \ldots, t_{n-1}\right) \tilde{H}_{k}\left(t_{n}\right) & (\text { for } n>1) .\end{cases}
$$

Since $\tilde{H}_{i}\left(t_{1}, \ldots, t_{n}\right)$ equals the $i$-th weighted complete symmetric polynomial $h_{i}^{\left\{a_{m}\right\}}\left(t_{1}, \ldots, t_{n}\right)$, we have the Eq. (2).

We obtain the Eq. (4) from the Eq. (2) by applying $*$.

Since $D\left(t_{1}\right) \cdots D\left(t_{n}\right)$ and $U\left(t_{n}\right) \cdots U\left(t_{1}\right)$ are generalized Schur operators with $\left\{a_{m}\right\}$, $U^{*}\left(t_{1}\right) \cdots U^{*}\left(t_{n}\right)$ and $D^{*}\left(t_{n}\right) \cdots D^{*}\left(t_{1}\right)$ are also generalized Schur operators with $\left\{a_{m}\right\}$. Applying the Eq. (4) Proposition 3.1 to $U^{*}\left(t_{1}\right) \cdots U^{*}\left(t_{n}\right)$ and $D^{*}\left(t_{n}\right) \cdots D^{*}\left(t_{1}\right)$, we obtain the Eqs. (3) and (5), respectively.

Hence Proposition 3.1 follows.

\section{More examples}

In this section, we consider some examples of generalized Schur operators.

\subsection{Shifted shapes}

This example is the same as [5, Example 2.1]. Let $Y$ be the set of shifted shapes, i.e.,

$$
Y=\left\{\left\{(i, j) \in \mathbb{N}^{2} \mid i \leq j \leq \lambda_{i}+i\right\} \mid \lambda=\left(\lambda_{1}>\lambda_{2}>\cdots\right), \lambda_{i} \in \mathbb{N}\right\} .
$$

For $\lambda \subset v \in Y$, let $c c_{0}(\lambda \backslash v)$ denote the number of connected components of $\lambda \backslash v$ that do not intersect with the main diagonal, and $\operatorname{cc}(\lambda \backslash \nu)$ the number of connected components of $\lambda \backslash v$. For example, let $\lambda=(7,5,3,2)$ and $\mu=(5,4,2)$. In this case, $\lambda \backslash v$ is the set of boxes $\circ$ and $\bullet$ in

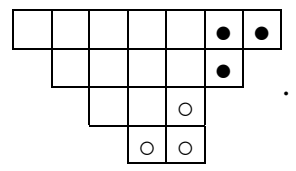

Since the component of the boxes $\circ$ intersects with the main diagonal at $(4,4), \operatorname{cc}_{0}(\lambda \backslash$ $v)=1$ and $c c(\lambda \backslash \nu)=2$. 
For $\lambda \in Y, D_{i}$ are defined by

$$
D_{i} \lambda=\sum_{\nu} 2^{c c_{0}(\lambda \backslash \nu)} v
$$

where the sum is over all $\nu$ 's that are obtained from $\lambda$ by removing $i$ boxes, with no two box in the same diagonal.

For $\lambda \in Y, U_{i}$ are defined by

$$
U_{i} \lambda=\sum_{\mu} 2^{c c(\mu \backslash \lambda)} \mu
$$

where the sum is over all $\mu$ 's that are obtained from $\lambda$ by adding $i$-boxes, with no two box in the same diagonal.

In this case, since $D(t)$ and $U(t)$ satisfy

$$
D\left(t^{\prime}\right) U(t)=\frac{1+t t^{\prime}}{1-t t^{\prime}} U(t) D\left(t^{\prime}\right)
$$

$D\left(t_{1}\right) \cdots D\left(t_{n}\right)$ and $U\left(t_{n}\right) \cdots U\left(t_{1}\right)$ are generalized Schur operators with $\{1,2,2,2, \ldots\}$. (See [5].) In this case, for $\lambda, \mu \in Y$, generalized Schur polynomials $s_{\lambda, \mu}^{D}$ and $s_{U}^{\lambda, \mu}$ are respectively the shifted skew Schur polynomials $Q_{\lambda / \mu}\left(t_{1}, \ldots, t_{n}\right)$ and $P_{\lambda / \mu}\left(t_{1}, \ldots, t_{n}\right)$.

In this case, Proposition 3.5 reads as

$$
h_{i}^{\{1,2,2,2, \ldots\}}\left(t_{1}, \ldots, t_{n}\right)=\left\{\begin{array}{ll}
2 Q_{(i)}\left(t_{1}, \ldots, t_{n}\right) & i>0 \\
Q_{\varnothing}\left(t_{1}, \ldots, t_{n}\right) & i=0
\end{array} .\right.
$$

It also follows from Proposition 3.10 that

$$
h_{i}^{\{1,2,2,2, \ldots\}}\left(t_{1}, \ldots, t_{n}\right)=P_{(i)}\left(t_{1}, \ldots, t_{n}\right) .
$$

Furthermore, Corollary 3.4 reads as

$$
\sum_{\mu} 2^{c c(\mu \backslash \lambda)} Q_{\mu}\left(t_{1}, \ldots, t_{n}\right)=h_{i}^{\{1,2,2,2, \ldots\}} Q_{\lambda}\left(t_{1}, \ldots, t_{n}\right),
$$

where the sum is over all $\mu$ 's that are obtained from $\lambda$ by adding $i$ boxes, with no two in the same diagonal.

\subsection{Young's lattice: dual identities}

This example is the same as [5, Example 2.4]. Let $Y$ be Young's lattice $\mathbb{Y}$, and $D_{i}$ the same ones in the prototypical example, (i.e., $D_{i} \lambda=\sum_{\mu} \mu$, where the sum is over all $\mu$ 's that are obtained from $\lambda$ by removing $i$ boxes, with no two in the same column.) For $\lambda \in Y, U_{i}^{\prime}$ are defined by $U_{i}^{\prime} \lambda=\sum_{\mu} \mu$, where the sum is over all $\mu$ 's that are 
obtained from $\lambda$ by adding $i$ boxes, with no two in the same row. (In other words, $D_{i}$ removes horizontal strips, while $U_{i}^{\prime}$ adds vertical strips.)

In this case, since $D(t)$ and $U^{\prime}(t)$ satisfy

$$
D(t) U^{\prime}\left(t^{\prime}\right)=\left(1+t t^{\prime}\right) U^{\prime}\left(t^{\prime}\right) D(t)
$$

$D\left(t_{1}\right) \cdots D\left(t_{n}\right)$ and $U^{\prime}\left(t_{n}\right) \cdots U^{\prime}\left(t_{1}\right)$ are generalized Schur operators with $\{1,1,0,0,0, \ldots\}$. (See [5].) In this case, for $\lambda, \mu \in Y$, generalized Schur polynomials $s_{U^{\prime}}^{\lambda, \mu}$ equal $s_{\lambda^{\prime} / \mu^{\prime}}\left(t_{1}, \ldots, t_{n}\right)$, where $\lambda^{\prime}$ and $\mu^{\prime}$ are the transposes of $\lambda$ and $\mu$, and $s_{\lambda^{\prime} / \mu^{\prime}}\left(t_{1}, \ldots, t_{n}\right)$ are skew Schur polynomials.

In the prototypical example (Example 3.6), Corollary 3.4 is the classical Pieri's formula, the formula describing the product of a complete symmetric polynomial and a Schur polynomial. In this example, Corollary 3.4 is the dual Pieri's formula, the formula describing the product of a elementary symmetric polynomial and a Schur polynomial.

In this case, Corollary 3.5 reads as

$$
h_{i}^{\{1,1,0,0,0, \ldots\}}\left(t_{1}, \ldots, t_{n}\right)=s_{\left(1^{i}\right)}\left(t_{1}, \ldots, t_{n}\right)=e_{i}\left(t_{1}, \ldots, t_{n}\right),
$$

where $e_{i}\left(t_{1}, \ldots, t_{n}\right)$ denotes the $i$-th elementally symmetric polynomials.

Furthermore, Corollary 3.4 reads as

$$
\sum_{\mu} s_{\mu}\left(t_{1}, \ldots, t_{n}\right)=e_{i}\left(t_{1}, \ldots, t_{n}\right) s_{\lambda}\left(t_{1}, \ldots, t_{n}\right)
$$

where the sum is over all $\mu$ 's that are obtained from $\lambda$ by adding a vertical strip consisting of $i$ boxes.

For a skew Young diagram $\lambda / \mu$ and $i \in \mathbb{N}$, Theorem 3.2 reads as

$$
\sum_{\kappa} s_{\kappa / \mu}\left(t_{1}, \ldots, t_{n}\right)=\sum_{j=0}^{i} \sum_{\nu} e_{i-j}\left(t_{1}, \ldots, t_{n}\right) s_{\lambda / \nu}\left(t_{1}, \ldots, t_{n}\right)
$$

where the first sum is over all $\kappa$ 's that are obtained from $\lambda$ by adding a vertical strip consisting $i$ boxes; the last sum is over all $\nu$ 's that are obtained from $\mu$ by removing a vertical strip consisting $j$ boxes.

\subsection{Planar binary trees}

This example is the same as [7]. Let $F$ be the monoid of words generated by the alphabet $\{1,2\}$ and 0 denote the word of length 0 . We give $F$ the structure of a poset by $v \leq v w$ for $v, w \in F$. We call an ideal of the poset $F$ a planar binary tree or shortly a tree. An element of a tree is called a node of the tree. We write $\mathbb{T}$ for the set of trees and $\mathbb{T}_{i}$ for the set of trees with $i$ nodes. We respectively call nodes $v 2$ and $v 1$ right and left children of $v$. A node without a child is called a leaf. For $T \in \mathbb{T}$ and $v \in F$, we define $T_{v}$ to be $\{w \in T \mid v \leq w\}$. 
First we define up operators. We respectively call $T^{\prime}$ a tree obtained from $T$ by adding some nodes right-strictly and left-strictly if $T \subset T^{\prime}$ and each $w \in T^{\prime} \backslash T$ has no right children and no left children. We define linear operators $U_{i}$ and $U_{i}^{\prime}$ on $K \mathbb{T}$ by

$$
\begin{aligned}
& U_{i} T=\sum_{T^{\prime}} T^{\prime}, \\
& U_{i}^{\prime} T=\sum_{T^{\prime \prime}} T^{\prime \prime},
\end{aligned}
$$

where the first sum is over all $T^{\prime}$ 's that are obtained from $T$ by adding $i$ nodes rightstrictly, and the second sum is over all $T^{\prime \prime}$ 's that are obtained from $T$ by adding $i$ nodes left-strictly. For example,

$$
\begin{aligned}
& U_{2}\{0\}=\{0,1,11\}+\{0,1,2\}+\{0,2,21\}, \\
& U_{2}^{\prime}\{0\}=\{0,2,22\}+\{0,1,2\}+\{0,1,12\} .
\end{aligned}
$$

Next we define down operators. For $T \in \mathbb{T}$, let $r_{T}$ be $\left\{w \in T \mid w 2 \notin T\right.$. If $w=v 1 w^{\prime}$ then $v 2 \notin T$. $\}$, i.e., the set of nodes which have no child on its right and which belong between 0 and the rightmost leaf of $T$. The set $r_{T}$ is a chain. Let $r_{T}=\left\{w_{T, 1}<w_{T, 2}<\right.$ $\cdots\}$. We define linear operators $D_{i}$ on $K \mathbb{T}$ by

$$
D_{i} T= \begin{cases}\left(\cdots\left(\left(T \ominus w_{T, i}\right) \ominus w_{T, i-1}\right) \cdots\right) \ominus w_{T, 1} & i \leq\left|r_{T}\right| \\ 0 & i>\left|r_{T}\right|\end{cases}
$$

for $T \in \mathbb{T}$, where

$$
T \ominus w=\left(T \backslash T_{w}\right) \cup\left\{w v \mid w 1 v \in T_{w}\right\}
$$

for $w \in T$ such that $w 2 \notin T$. Roughly speaking, $D_{i} T$ is the tree obtained from $T$ by evacuating the $i$ topmost nodes without a child on its right and belonging between 0 and the rightmost leaf of $T$. For example, let $T$ be $\{0,1,11,12,121\}$. Since $w_{T, 1}=0$, $w_{T, 2}=12$ and

$$
\{0,1,11,12,121\} \stackrel{\ominus 12}{\longrightarrow}\{0,1,11,12\} \stackrel{\ominus 0}{\longrightarrow}\{0,1,2\}
$$

we have $D_{2} T=\{0,1,2\}$.

These operators $D(t), U\left(t^{\prime}\right)$ and $U^{\prime}\left(t^{\prime}\right)$ satisfy the following equations:

$$
\begin{aligned}
D(t) U\left(t^{\prime}\right) & =\frac{1}{1-t t^{\prime}} U\left(t^{\prime}\right) D(t), \\
D(t) U^{\prime}\left(t^{\prime}\right) & =\left(1+t t^{\prime}\right) U^{\prime}\left(t^{\prime}\right) D(t) .
\end{aligned}
$$

(See [7] for a proof of the equations.) Hence the generalized Schur polynomials for these operators satisfy the same Pieri's formula as in the case of the classical Young's lattice and its dual construction. 
In this case, generalized Schur polynomials are not symmetric in general. For example, since

$$
\begin{aligned}
& D\left(t_{1}\right) D\left(t_{2}\right)\{0,1,12\} \\
& \quad=D\left(t_{1}\right)\left(\{0,1,12\}+t_{2}\{0,2\}+t_{2}^{2}\{0\}\right) \\
& \quad=\left(\{0,1,12\}+t_{1}\{0,2\}+t_{1}^{2}\{0\}\right)+t_{2}\left(\{0,2\}+t_{1}\{0\}\right)+t_{2}^{2}\left(\{0\}+t_{1} \emptyset\right),
\end{aligned}
$$

$s_{\{0,1,12\}, \emptyset}^{D}\left(t_{1}, t_{2}\right)=t_{1} t_{2}^{2}$ is not symmetric.

We define three kinds of labeling on trees to give generalized Schur polynomials $s_{U}^{T, \emptyset}\left(t_{1}, \ldots, t_{n}\right), s_{U^{\prime}}^{T, \emptyset}\left(t_{1}, \ldots, t_{n}\right)$ and $s_{T, \emptyset}^{D}\left(t_{1}, \ldots, t_{n}\right)$ presentations as generating functions of them.

Definition 4.1. Let $T$ be a tree and $m$ a positive integer. We call a map $\varphi: T \rightarrow$ $\{1, \ldots, m\}$ a right-strictly-increasing labeling if

- $\varphi(w) \leq \varphi(v)$ for $w \in T$ and $v \in T_{w 1}$ and

- $\varphi(w)<\varphi(v)$ for $w \in T$ and $v \in T_{w 2}$.

We call a map $\varphi: T \rightarrow\{1, \ldots, m\}$ a left-strictly-increasing labeling if

- $\varphi(w)<\varphi(v)$ for $w \in T$ and $v \in T_{w 1}$ and

- $\varphi(w) \leq \varphi(v)$ for $w \in T$ and $v \in T_{w 2}$.

We call a map $\varphi: T \rightarrow\{1, \ldots, m\}$ a binary-searching labeling if

- $\varphi(w) \geq \varphi(v)$ for $w \in T$ and $v \in T_{w 1}$ and

- $\varphi(w)<\varphi(v)$ for $w \in T$ and $v \in T_{w 2}$.

For example, let $T=\{0,1,2,11,21,22\}$. We write a labeling $\varphi$ on $T$ as the diagram

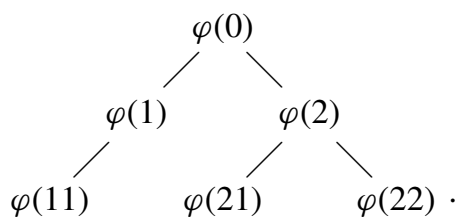

In this notation, the labelings

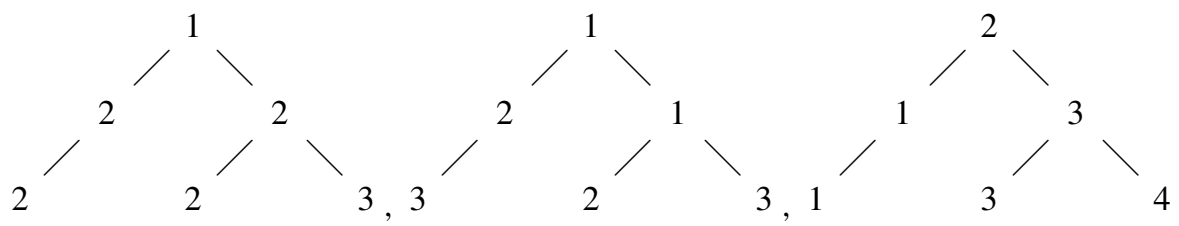

on $T$ are a right-strictly-increasing labeling, a left-strictly-increasing labeling and a binary-searching labeling, respectively.

The inverse image $\varphi^{-1}(\{1, \ldots, n+1\})$ of a right-strictly-increasing labeling $\varphi$ is the tree obtained from the inverse image $\varphi^{-1}(\{1, \ldots, n\})$ by adding some nodes Springer 
right-strictly. Hence we identify right-strictly-increasing labelings with sequences $\left(\emptyset=T^{0}, T^{1}, \ldots, T^{m}\right)$ of $m+1$ trees such that $T^{i+1}$ is obtained from $T^{i}$ by adding some nodes right-strictly for each $i$.

Similarly, we identify left-strictly-increasing labelings with sequences $(\emptyset=$ $\left.T^{0}, T^{1}, \ldots, T^{m}\right)$ of $m+1$ trees such that $T^{i+1}$ is obtained from $T^{i}$ by adding some nodes left-strictly for each $i$.

For a binary-searching labeling $\varphi_{m}: T \rightarrow\{1, \ldots, m\}$, by the definition of binarysearching labeling, the inverse image $\varphi_{m}^{-1}(\{m\})$ equals $\left\{w_{T, 1}, \ldots, w_{T, k}\right\}$ for some $k$. We can obtain a binary-searching labeling $\varphi_{m-1}: T \ominus \varphi_{m}^{-1}(\{m\}) \rightarrow\{1, \ldots, m-1\}$ from $\varphi_{m}$ by evacuating $k$ nodes $\varphi_{m}^{-1}(\{m\})$ together with their labels. Hence we identify binary-searching labelings with sequences $\left(\emptyset=T^{0}, T^{1}, \ldots, T^{m}\right)$ of $m+1$ trees such that $D_{k_{i}} T^{i}=T^{i-1}$ for some $k_{1}, k_{2}, \ldots, k_{m}$.

For a labeling $\varphi$ from $T$ to $\{1, \ldots, m\}$, we define $t^{\varphi}=\prod_{w \in T} t_{\varphi(w)}$. For a tree $T$, it follows that

$$
\begin{aligned}
& s_{U}^{T, \emptyset}\left(t_{1}, \ldots, t_{n}\right)=\sum_{\varphi} t^{\varphi}, \\
& s_{U^{\prime}}^{T, \emptyset}\left(t_{1}, \ldots, t_{n}\right)=\sum_{\phi} t^{\phi}, \\
& s_{T, \emptyset}^{D}\left(t_{1}, \ldots, t_{n}\right)=\sum_{\psi} t^{\psi},
\end{aligned}
$$

where the first sum is over all right-strictly-increasing labelings $\varphi$ on $T$, the second sum is over all left-strictly-increasing labelings $\phi$ on $T$, and the last sum is over all binary-searching labelings $\psi$ on $T$.

\section{References}

1. Bergeron, Mykytiuk, Sottile, and van Willigenburg, "Non-commutative Pieri operators on posets," $J$. Combin. Th. Ser. A 91 (2000), 84-110.

2. S. Fomin, "Generalized Robinson-Schensted-Knuth correspondence," Zariski Nauchn. Sem. Leningrad. Otdel. Mat. Inst. Steklov. (LOMI) 155 (1986), 156-175, 195 (Russian); English transl., J. Soviet Math. 41 (1988), 979-991.

3. S. Fomin, "Duality of graded graphs," J. Algebraic Combin. 3 (1994), 357-404.

4. S. Fomin, "Schensted algorithms for dual graded graphs," J. Algebraic Combin. 4 (1995), 5-45.

5. S. Fomin, "Schur operators and Knuth correspondences," J. Combin. Theory Ser. A 72 (1995), 277-292.

6. T. Lam, "A combinatorial generalization of the Boson-Fermion correspondence," Math. Res. Lett. 13(3) (2006), 377-329.

7. Y. Numata, "An example of generalized Schur operators involving planar binary trees," preprint, arXiv:math. CO/0609376.

8. T. Roby, "Applications and extensions of Fomin's generalization of the Robinson-Schensted correspondence to differential posets," Ph.D. thesis, M.I.T., 1991.

9. R. Stanley, "Differential posets, J. Amer. Math. Soc., 1 (1988), 919-961.

10. R. Stanley, "Variations on differential posets," in D. Stanton, (ed.), Invariant Theory and Tableaux IMA volumes in mathematics and its applications, Springer-Verlag, New York, pp. 145-165. 